\title{
Local Group Velocity from the PSCz and BTP Surveys
}

\author{
Kenton J. D'Mellow ${ }^{\mathrm{A}}$, Will Saunders ${ }^{\mathrm{B}, \mathrm{C}}$, and PSCz/BTP Teams

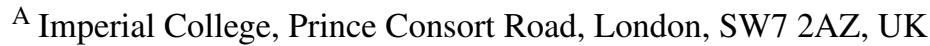 \\ ${ }^{B}$ Anglo-Australian Observatory, Epping NSW 1710, Australia \\ ${ }^{\mathrm{C}}$ Corresponding author. Email: will@ aaoepp.aao.gov.au
}

Received 2004 May 3, accepted 2004 November 15

\begin{abstract}
We present a brief overview and preliminary measure of the Local Group velocity, using the $\mathrm{PSCz}$ survey together with its recently completed extension into the Galactic plane, the Behind The Plane (BTP) survey. The addition of the BTP has increased the total sky coverage from $84 \%$ to $93 \%$, drastically reducing the systematic uncertainty in the direction of the local gravitational pull caused by incomplete sky coverage. We present methods that self-consistently determine the acceleration in the presence of redshift distortions. Preliminary results suggest that the dipole converges within the survey limiting depth. There is a large, but only marginally significant, component to the dipole arising at $180-200 h^{-1} \mathrm{Mpc}$.
\end{abstract}

Keywords: cosmology: large-scale structure — galaxies: kinematics and dynamics

\section{Behind The Plane Extension Survey}

The BTP is the low-latitude extension to the PSCz (Point Source Catalogue redshift) survey (Saunders et al. 2000a) based on the IRAS PSC, which mapped the entire sky at $12,25,60$, and $100 \mu \mathrm{m}$, with nearly uniform all-sky coverage - ideal as an all-sky redshift catalogue sample base. The IRAS selection criteria were tightened for the BTP, in order to minimise contamination from Galactic sources. These criteria were $S_{60}>2 S_{25}$ and $S_{60}>4 S_{12}$ to exclude stars, and $S_{100}<5 S_{60}$ to exclude Galactic cirrus. Also excluded were areas with $<2$ HCONS, areas of high source density at 12,25 , and $60 \mu \mathrm{m}$, and areas with $I_{100}>25 \mathrm{MJy} \mathrm{sr}^{-1}$. The remaining unmapped sky is due to the IRAS coverage gaps (3\%) and the ZOA (4\%). The survey has a median depth of $8500 \mathrm{~km} \mathrm{~s}^{-1}$ with low and quantified incompleteness. The BTP consists of 1225 PSC likely or confirmed galaxies, of which 869 have positive IDs and redshifts. Completeness issues are briefly described in Saunders et al. (2000b), and will be covered in detail in the official survey release (Saunders et al., in prep.).

\section{Local Group Velocity}

Here we describe the problems created when using a magnitude-limited redshift sample to measure the Local Group velocity $\mathbf{v}_{\mathrm{LG}}$. Linear gravitational instability theory states that the velocity vector can in principle, be determined by integrating the gravitational force from the density field over the entire volume of the Universe (Peebles 1980). In reality we can make an estimate of this, using a sparse sample of the density field over a sufficiently large, finite volume. By using luminous galaxies as a tracer of the underlying mass, we get

$$
\mathbf{v}_{\mathrm{LG}}=\frac{H_{0} \beta}{4 \pi} \sum_{\text {galaxies }} \frac{w(\mathbf{r}) \hat{\mathbf{r}}}{\psi(\mathbf{r})\left|\mathbf{r}^{2}\right|}
$$

Here, $\beta=\Omega^{0.6} / b$, where $b$ is the bias of the galaxy population under consideration, and $\psi(\mathbf{r})$ is the selection function - the expected number density of galaxies (in the absence of clustering) meeting the survey selection criteria, as a function of position (Strauss et al. 1992). The issue being that we have a redshift-space sample, but want real-space distances to each galaxy. For many cosmological statistics, these redshift-space distortions are critical. Conversely, the Local Group velocity is only sensitive to first-order changes in the mass distribution: non-linear distortions, causing the misshapen appearance of clusters, only affects the above equation to second order. The main advantage of this insensitivity is that the predominant concern is only that the calculation should be performed in the correct frame of reference. The velocity field is coherent over small scales, so nearby mass should be considered in the Local Group frame of reference. At greater distances, the cosmic microwave background (CMB) frame of reference is necessary. Choosing, a priori, a transition between frames of reference is a rather arbitrary and essentially non-optimal approach.

\subsection{Self-Consistent Iterative Schemes}

We can self-consistently determine the transfer function by first considering the gravitational acceleration we experience due to our closest neighbours, and then altering the frame of reference accordingly before looking at more distant sources. This is based on the assumption that the motion of the Local Group is entirely due to galaxies 
from the volume interior to the considered distance. The next-closest source is now corrected with respect to this reference frame. This way, a smooth transition between working in the Local Group frame, to working in the CMB frame is achieved during the calculation. The resultant equation pair is

$$
\mathbf{v}=\frac{H_{0} \beta}{4 \pi} \sum_{i=1}^{N} \frac{\hat{\mathcal{R}}_{i} w\left(\mathcal{R}_{i}, k_{c}\right)}{\psi\left|\mathcal{R}_{i}^{2}\right|}
$$

where the corrected source distance is given by

$$
\mathcal{R}_{i}=\mathbf{r}_{i}+\hat{\mathbf{r}}_{i} \cdot \frac{\beta_{\mathrm{old}}}{4 \pi} \sum_{j=1}^{i-1} \frac{\mathcal{A}_{j} \hat{\mathcal{R}}_{j} w\left(\mathcal{R}_{j}, k_{c}\right)}{\psi\left|\mathcal{R}_{j}^{2}\right|}
$$

Here, $\beta$, and $\beta_{\text {old }}$ indicate two consecutive evaluations of $\beta$ across iterations of the calculation. At the calculation limit, $\beta$ can be determined by normalisation of the velocity magnitude to that of the CMB temperature dipole. Convergence is reached when the system achieves consistency in $\beta$. Systems such as this can suffer from the rocket effect, or Kaiser effect (Kaiser 1987), resulting in divergent behaviour but this can be tempered by dampening $\beta$ between iterations, in a similar manner to that of Strauss et al. (1992). We find that by doing this, convergence is consistently achieved within of order ten iterations. Using this method, one allows the reference frame transfer function to be completely described by the data, and enables the simultaneously determination of the velocity direction, $\beta$, and LG transfer function. Results are shown on the left hand side of Figure 1.

\subsection{PIZA Reconstruction}

Valentine et al. (2000) applied the Path Interchange Zel'dovich Approximation (PIZA) reconstruction method
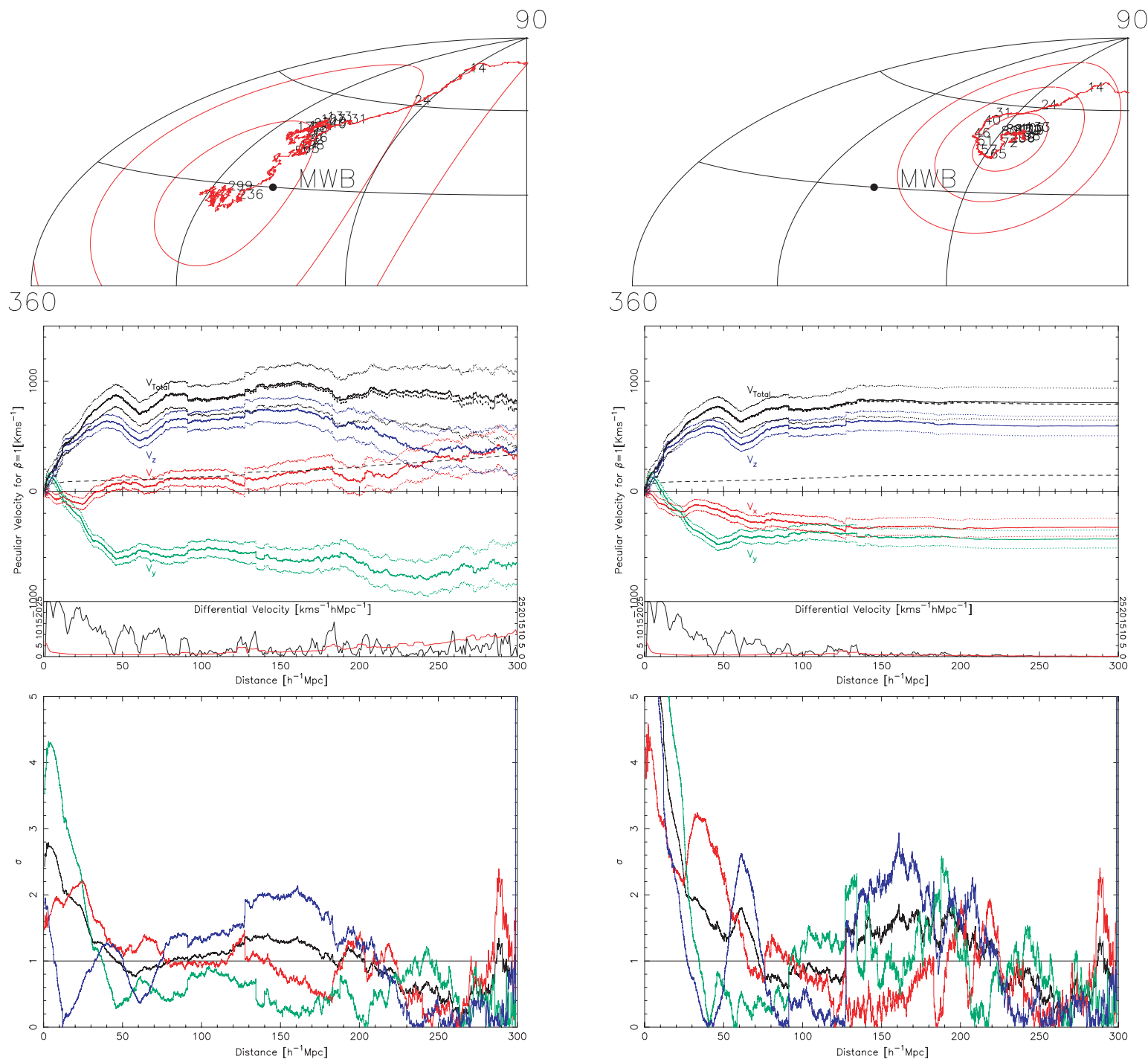

Figure 1 Dipole calculations for PCSz+BTP: (left) unweighted and (right) with minimum variance weighting. Top: Dipole direction and direction path as a function of included survey depth, with 1-, 2-, and $3 \sigma$ Poisson errors around the final direction. Centre: Velocity components as a function of distance, and component influence over that expected from Poisson noise. Bottom: Significance of dipole contribution, over the expected shot noise, from beyond the given distance. Non-PIZA data is treated using the self-consistenent iterative dipole method. In all component figures, $V_{x}, V_{y}, V_{z}$, and $V_{\text {total }}$ are in red, green, blue, and black respectively. Component error bars are $1 \sigma$, based on Poisson noise. 
to the PSCz, a least-action principle based algorithm first proposed by Croft \& Gaztañaga (1997). As an initial trial, the PIZA reconstructed volumes of Valentine et al. have also been used in calculation of the Local Group velocity, alongside unreconstructed BTP data treated as per the above iterative scheme. This serves as a good indicator of the dipole, only biased by residual redshift-space distortions on a small part of the dataset. The results are shown on the right hand side of Figure 1. An extension to the work of Valentine et al. to apply PIZA to the PSCz together with the recently completed BTP dataset will be completed shortly, thus allowing a full real-space dipole estimate.

\section{Results and Discussion}

The dipole is calculated without and with the minimum variance weighting of Strauss et al. (1992). The unweighted sum is our best estimate of the dipole in the absence of any other knowledge of cosmology, and is formally divergent at large distances. The weighted sum is our best estimate for the dipole, given what we know about the clustering of these galaxies. Results from these calculations are given in Table 1. There are two salient features to note. Firstly, the alignment is quite poor when using minimum variance weighting, but astoundingly good when no weighting is used. Secondly, there is a significant change in all three components of the velocity vector between 180 and $200 h^{-1} \mathrm{Mpc}$.

\subsection{Effect of the Minimum Variance Weighting}

Strauss' optimal weighting scheme gives a cutoff scale dependent upon $P(k)$ and sampling. When employed using the PSCz turnover scale $0.25 \leq k_{\mathrm{c}} \leq 0.35$ (Saunders et al. 2000a) it cuts the influence around $150 \mathrm{~h}^{-1} \mathrm{Mpc}$. This leaves the velocity direction stranded at high Galactic latitude, giving poor alignment. In contrast, the unweighted solutions allow influence from around $200 \mathrm{~h}^{-1} \mathrm{Mpc}$, pulling the dipole down to lower latitudes and resulting in much better directional agreement with the CMB. It is important to remember that the weighting scheme is designed explicitly to minimise the expected

Table 1. Dipole alignments and $\beta_{I R A S}$ estimates calculated unweighted and with minimum variance $J_{3}$ weighting. Note that projected $\Omega_{m}$ values are given based on the assumption that $\beta_{\text {IRAS }}=1.3^{-1}$, as consistent with recent estimates. Note also poor alignment, and high $\beta_{\text {IRAS }}$ estimates when using $J_{3}$ weighting

\begin{tabular}{lccc}
\hline Dataset & $\mathrm{CMB}$ misalignment $\left[^{\circ}\right]$ & $\beta_{I R A S}$ & $\Omega_{m}$ \\
\hline PIZA PSCz+BTP & 1.53 & 0.74 & 0.39 \\
PIZA PSCz+BTP+ $J_{3}$ & 28.1 & 0.63 & 0.30 \\
\hline
\end{tabular}

error given a well-defined sample of a representative portion of the Universe, and is not guaranteed to yield the minimum error in our exact local Universe.

\subsection{Influence at $180-200 \mathrm{~h}^{-1} \mathrm{Mpc}$}

In the unweighted sum, is apparent that there is significant influence on the Local Group velocity out to $\sim 200 \mathrm{~h}^{-1} \mathrm{Mpc}$. The statistical significance of contributions to the dipole from beyond a given depth, is also shown in Figure 1 (at the bottom). The contribution at 180-200 $h^{-1} \mathrm{Mpc}$ is marginally statistically significant. In physical terms, it amounts to a position-dependent deficit of about 40 galaxies out of approximately 100 expected in the redshift interval. At this distance both PSCz and BTP datasets are very sparse, disallowing an obvious visual identification of the structures responsible. However, the vectorial change in the dipole over this range is in the direction $[l, b] \sim[330,0]-$ in the direction of (but much more distant than) the Great Attractor.

\section{Preliminary Conclusions}

The dipole growth has shown some remarkable features at and around $200 \mathrm{~h}^{-1} \mathrm{Mpc}$ - indicative of a concentration on significant extent - but these have yet to be studied in greater detail. Beyond $200 h^{-1} \mathrm{Mpc}$, no further influential structure is indicated, and the residual drift of the velocity components is easily explained by Poisson noise from the increasingly sparse outer regions. This suggests that the dipole has converged by this depth, in agreement with the findings of Rowan-Robinson et al. (2000).

\section{References}

Croft, R. A. C., \& Gaztañaga, E. 1997, MNRAS, 285, 793

Kaiser, N. 1987, MNRAS, 227, 1

Peebles, P. J. E. 1980, The Large-Scale Structure of the Universe (Princeton: Princeton University Press)

Rowan-Robinson, M., Sharpe, J., Oliver, S. J., Keeble, O., Canavazes, A., Saunders, W., Taylor, A. N., Valentine, H., Frenk, C. S., Efstathiou, G. P., McMahon, R. G., White, S. D. M., Sutherland, W. J., Tadros, H., \& Maddox, S. J. 2000, MNRAS, 314,375

Saunders, W., Sutherland, W. J., Maddox, S. J., Keeble, O., Oliver, S. J., Rowan-Robinson, M., McMahon, R. G., Efstathiou, G. P., Tadros, H., White, S. D. M., Frenk, C. S., Carramiñana, A., \& Hawkins, M. R. S. 2000a, MNRAS, 317, 55

Saunders, W., D’Mellow, K. J., Tully, R. B., Mobasher, B., Maddox, S., Sutherland, W., Carrasco, E., Clements, D., \& Staveley-Smith, L. 2000b, ASP Conf. Ser. 201, eds. S. Courteau, M. A. Strauss, \& J. A. Willick (San Francisco: ASP), 237

Saunders, W., D’Mellow, K. J., Tully, R. B., Mobasher, B., Maddox, S., Sutherland, W., Carrasco, E., Clements, D., \& Staveley-Smith, L., in prep.

Strauss, M. A., Yahil, A., Davis, M., Huchra, J. P., \& Fisher, K. 1992, ApJ, 397, 395

Valentine, H., Saunders, W., \& Taylor, A. 2000, MNRAS, 319, 13 